\section{Detection of rotavirus in handwashings of attendants of children with diarrhoea}

Rotavirus diarrhoea is common in infants and young children and appears to have a worldwide distribution. While faecal-oral route of transmission has been reported, ${ }^{1}$ potential vehicles of transmission are not known. Nosocomially acquired outbreaks of rotavirus diarrhoea have occurred in nurseries ${ }^{2}$ and paediatric wards ${ }^{3}$ in developed countries. None the less, we have detected rotavirus in handwashings of attendants of patients with rotavirus diarrhoea more often than in those of attendants of children with non-rotavirus diarrhoea.

\section{Patients, methods, and results}

The subjects for the study comprised 147 children aged under 5 years admitted to this centre for treatment of diarrhoea. Selection was based on inclusion of the first four children admitted each day. Stools were collected on admission for detection of rotavirus.

Within four hours of these admissions the hands of all attendants were washed thoroughly in a sterilised bowl with $100 \mathrm{ml}$ tap water and the washings examined for rotavirus. A $20 \%$ sample of tap water used for washings was sampled at random also for detection of rotavirus. The handwashing water and sampled tap water were concentrated by an adaptation of the polyethylene glycol hydroextraction method, ${ }^{4}$ in which handwashings were poured into a $30 \mathrm{~cm}$ dialysing tube $3.2 \mathrm{~cm}$ wide (MW Cutoff 12000 14000) and knotted for closure. After completely covering with polyethylene glycol 4000 the tube was stored at $4^{\circ} \mathrm{C}$ overnight during which time water was completely extracted. The tube was then washed thoroughly with running tap water to remove the polyethylene glycol from its outer surface, cut $1 \mathrm{~cm}$ below the knot, and $2.5 \mathrm{ml}$ tryptose phosphate broth introduced into it. The open end of the tube was securely held between the fingers and shaken completely to wet the inside surface with the broth. The concentration was then passed through a $0.2 \mu \mathrm{m}$ membrane filter (Millipore) and the filtrate stored at $4^{\circ} \mathrm{C}$ until tested for rotavirus. Stool specimens and handwashing concentrates were tested for rotavirus antigen using a confirmatory ELISA test, in which plates were precoated in alternate wells with rotavirus immune and non-immune sera of the same goat. ${ }^{5}$ All laboratory assays were performed blind.

Rotavirus diarrhoea was more common among children aged 3-23 months. Handwashings of $78.6 \%$ of the attendants of patients $(n=70)$ with rotavirus diarrhoea and $19.5 \%$ of attendants of patients with non-rotavirus diarrhoea $(n=77)$ were positive for rotavirus antigen $(p<0.001)$. The rate of detection of rotavirus in handwashings of attendants of patients with rotavirus diarrhoea was significantly higher in younger children (0-23 months) than in older children ( $24-59$ months) $(p<0.02$; table). None of the samples of tap water contained rotavirus.

Detection of rotavirus from handwashing of attendants of children of various ages with diarrhoea

\begin{tabular}{|c|c|c|c|c|}
\hline \multirow[b]{2}{*}{$\begin{array}{c}\text { Age } \\
\text { (months) }\end{array}$} & \multirow[b]{2}{*}{$\begin{array}{l}\text { Rotavirus in } \\
\text { handwashing }\end{array}$} & \multicolumn{2}{|c|}{ Attendants } & \multirow[b]{2}{*}{$\mathrm{p}$ Value } \\
\hline & & $\begin{array}{l}\text { Rotavirus } \\
\text { diarrhoea }\end{array}$ & $\begin{array}{c}\text { Non-rotavirus } \\
\text { diarrhoea }\end{array}$ & \\
\hline $0-23$ & $\left\{\begin{array}{l}+ \\
-\end{array}\right.$ & $\begin{array}{l}50 \\
9^{(a)}\end{array}$ & $\begin{array}{l}12 \text { (c) } \\
37^{(c)}\end{array}$ & (a) $v$ (c) $\mathrm{p}<0.001^{*}$ \\
\hline 24-59 & $\left\{\begin{array}{l}+ \\
-\end{array}\right.$ & ${ }_{6}^{5}{ }^{(b)}$ & ${ }_{25}^{3}(d)$ & (b) $v$ (d) $\mathrm{p}=0.027 \dagger$ \\
\hline
\end{tabular}

+ $2 \times 2 \chi^{2}$ test.

\section{Comment}

This study shows that rotavirus can be detected by a simple method from handwashings of attendants of patients. The high rate of detection in attendants of patients with rotavirus suggests that the method is a sensitive indicator of faecal contamination of hands and may be useful in future studies to trace rotavirus transmission. That rotavirus was found in the hand washings of $19.5 \%$ of attendants of patients with non-rotavirus diarrhoea suggests that they may have come into contact with other attendants and patients in adjacent beds. This implicates a possible role for hands in the spread of rotavirus infection. Hospital outbreaks of rotavirus infections ${ }^{2} 3$ might have been due to contamination of hands.

The detection rate of rotavirus from the handwashings of attendants of younger children with rotavirus diarrhoea was higher than that in handwashings of attendants of older children. This may be due to the closer contact of attendants with younger children. Alternatively younger children may excrete more virus than older children, since the older children might be partially immune from an earlier infection.

${ }^{1}$ World Health Organisation sub-group of the Scientific Working Group on Epidemiology and Etiology. Rotavirus and other viral diarrhoeas. Geneva : WHO, 1979. (WHO/DDC/EPE/79.2.)

${ }^{2}$ Bishop RF, Hewstone AS, Davidson GP, Townley RRW, Holmes IH, Ruck BJ. An epidemic of diarrhoea in human neonates involving a rotavirus-like agent and "enteropathogenic" serotypes of Escherichia coli. F Clin Pathol 1976;29:46-9.

${ }^{3}$ Ryder RW, McGown JE, Hatch MH, Palmer EL. Rotavirus-like agent as a cause of nosocomial diarrhoea in infants. $\mathcal{F}$ Pediatr 1977;90:698-702.

4 Ramia S, Sattar SA. Second-step concentration of viruses in drinking and surface waters using polyethylene glycol hydroextraction. Can f Microbiol 1979;25:587-92.

${ }^{5}$ Kapikian AZ, Yolken RH, Greenberg HB, et al. Diagnostic procedures for viral, rickettsial and chlamydial infections. 5th ed. Washington DC: American Public Health Association, 1979.

(Accepted 6 October 1982)

International Centre for Diarrhoeal Disease Research, Bangladesh (ICDDR,B), GPO Box 128, Dacca-2, Bangladesh

AZIZ R SAMADI, MD, DPH, head, disease transmission programme MOHAMMAD I HUQ, DBACT, PHD, head, microbiology branch QUAZI S AHMED, MSC, senior research officer

\section{Reactive arthritis associated with Staphylococcus epidermidis peritonitis in patient undergoing continuous ambulatory peritoneal dialysis}

The term reactive arthritis was introduced by Aho $e t a^{1}$ to describe several forms of joint disease associated with infection elsewhere in the body. We describe a patient undergoing treatment for renal failure by continuous ambulatory peritoneal dialysis who developed arthritis after an episode of peritoneal infection caused by Staphylococcus epidermidis, a bacterium not previously described as an aetiological agent in reactive arthritis.

\section{Case report}

A 62 year old man with end-stage renal failure secondary to hypertension was started on continuous ambulatory peritoneal dialysis in July 1981. Fou months later a peritoneal infection with $S$ epidermidis was treated successfull with cefuroxime. Over the next six months he had four further episodes of abdominal pain and cloudy peritoneal dialysis fluid. No organisms were isolated from the peritoneal fluid, but the symptoms and signs resolved after a short course of antibiotics on each occasion.

In March 1982 he had a further episode of abdominal pain and cloudy peritoneal fluid. Culture of dialysis fluid grew $S$ epidermidis, and a course of intravenous and intraperitoneal cefuroxime was given. Again the symptoms and signs of the peritoneal infection resolved. Three weeks later he began to complain of pain in the neck, shoulders, elbows, wrists, and lumbar region, and subsequently of pain and swelling of all the metacarpophalangea and proximal interphalangeal joints of both hands. There was no history of diarrhoea or urethritis, and the skin, mucous membranes, penis, and eyes were normal.

Investigations showed erythrocyte sedimentation rate $100 \mathrm{~mm}$ in the firs hour, later falling to 5 ; haemoglobin concentration $8.5 \mathrm{~g} / \mathrm{dl}$; white cell coun $11.4 \times 10^{\circ} / 1$; calcium concentration $2.53 \mathrm{mmol} / 1$ (10 mg/100 ml); phosphorus concentration $1.32 \mathrm{mmol} / 1(4 \mathrm{mg} / 100 \mathrm{ml})$; and alkaline phosphatase activity 201 IU/1. Blood and stool cultures gave negative results for Shigella, Salmonella, and Campylobacter. Rheumatoid and antinuclear factors were not 
detected; serum complement concentrations, DNA binding, and urate concentration were within the normal range; a test for hepatitis B surface antigen was negative. Radiography of the hands, neck, and sacroiliac joints showed no abnormality. Tissue type was HLA-B27.

He was treated with indomethacin $50 \mathrm{mg}$ twice daily. One week later the Silastic peritoneal catheter (Tenckhoff) was replaced. Three weeks later he had an upper gastrointestinal haemorrhage from an acute duodenal ulcer. This did not respond to conservative treatment, and vagotomy and.pyloroplasty were performed. He had an uneventful postoperative course, though the arthritis persisted for a further week.

Two months later he was still undergoing continuous ambulatory peritoneal dialysis and had had no further episodes of peritoneal infection or acute arthritis.

\section{Comment}

The clinical features in this case suggest that the polyarthritis was reactive to the $S$ epidermidis peritoneal infection. The arthritis started three weeks after the onset of peritonitis and was self-limiting, resolving spontaneously after five weeks. Other known causes of acute arthritis were excluded, and there was no evidence of infection with organisms more commonly associated with reactive arthritis. Furthermore, this patient was positive for HLA-B27, and such individuals are more at risk from this complication. ${ }^{1}$ We know of no other reports of reactive arthritis associated with $S$ epidermidis infection, though $S$ aureus is arthritogenic in the rat. ${ }^{2}$ With increasing numbers of patients undergoing continuous ambulatory peritoneal dialysis such a complication may be diagnosed more often in the future.

We thank Dr A J Rees for allowing us to report this case.

' Aho K, Ahvonen P, Lassus A, Siever K, Tiilikainen K. HLA-antigen 27 and reactive arthritis. Lancet 1973 ;ii:157.

${ }^{2}$ Hadler NM, Granovetter DA. Phlogistic properties of bacterial debris. Semin Arthritis Rheum 1978;8:1-16.

(Accepted 12 October 1982)

Renal Unit, Royal Postgraduate Medical School, Hammersmith Hospital, London W12 0HS

B R HUGHES, MB, CHB, senior house officer

C R $\mathbf{K}$ HIND, BSC, MRCP, registrar

\section{Lithium-induced constructional dyspraxia}

Lithium has a narrow therapeutic range, and the likelihood of serious neurotoxicity and encephalopathy increases as blood concentrations rise above $1.4 \mathrm{mmol}(\mathrm{mEq}) / 1$. After '14 days' dosage within the therapeutic range the drug causes subtle cognitive impairment in normal volunteers, ${ }^{1}$ but evidence from controlled studies of chronic administration in patients with manic depression, though conflicting, ${ }^{23}$ generally suggests that any cognitive changes at normal doses are not clinically important. We report here a case of constructional dyspraxia occurring at lithium concentrations within the therapeutic range after a year's previously uneventful administration.

\section{Case report}

A 50 year old housewife presented with endogenous depression of two years' duration. She had had episodes lasting about three months yearly for the previous five years. When first seen she was taking amitriptyline $150 \mathrm{mg}$ a day. The total plasma amitriptyline and nortriptyline concentration of $231 \mu \mathrm{g} / \mathrm{l}$ was possibly just above the therapeutic range, but reducing the dose to $125 \mathrm{mg}$ a day did not produce any improvement.

Amitriptyline was stopped and lithium carbonate started, but this did not produce a sustained remission. Thereafter she responded to combined amitriptyline $125 \mathrm{mg}$ a day and lithium $600 \mathrm{mg}$ a day. After remaining well and taking both drugs for the succeeding 14 months she began to complain of difficulties in finding words in conversation and of memory impairment, in the absence of any recurrence of depressive symptoms. Serum thyroxine concentration and free thyroxine index were normal, as was an electroencephalogram. Her 12-hour lithium concentration was $0.80 \mathrm{mmol} / 1$ and during the previous 14 months had ranged from 0.66 to $0.89 \mathrm{mmol} / 1$ Psychological testing showed a Mill Hill verbal IQ of 108 and a progressive matrices performance IQ of 98 . Her logical memory, paired associate learning, and memory of an array of pictures were normal both on immediate recall and after 30 minutes' delay. She had difficulty in finding words in ordinary conversation but not on formal testing for aphasia. A striking abnormality was her inability to copy the Rey picture (score on standard scoring criteria 14 out of 36 ). These findings indicated appreciable constructional dyspraxia and slight dysphasia.

Lithium was stopped but amitriptyline continued. One month later her word-finding difficulties had disappeared and her copy of the Rey picture (alternative form) was considerably improved, her score being 36 out of 36 . There were no appreciable differences in the results of the other tests. She remained affectively well on amitriptyline alone at follow-up after six months.

\section{Comment}

These findings indicate slowly developing constructional dyspraxia and mild dysphasia with difficulties of subjective memory occurring at lithium concentrations below $1.0 \mathrm{mmol} / \mathrm{l}$ and during concomitant use of amitriptyline. The impairment resolved completely when lithium was stopped and the tricyclic drug continued. Although these side effects may have been due to an interaction of both drugs, lithium and a tricyclic antidepressant are commonly prescribed together and the combination is usually regarded as being safe.

The possibility of cognitive difficulties caused by chronic administration of lithium at concentrations within the therapeutic range has not been discussed in recent reviews of lithium. Cases such as that reported here may be uncommon but nevertheless have practical implications: clinicians unaware of reports of research and particularly those not using the drug frequently may mistake cognitive impairment caused by the drug for a relapse of depression or early dementia. In cases of doubt formal psychological testing and retesting after the drug has been stopped are indicated.

1 Judd LL, Hubbard B, Janowsky DS, Leighton YH, Takahashi II. The effect of lithium carbonate on the cognitive functions of normal subjects. Arch Gen Psychiatry 1977;34:355-7.

2 Telford $R$. Worrall EP. Cognitive functions in manic-depressives: effects of lithium and physostigmine. Br $\mathcal{F}$ Psychiatry 1978;133:424-8.

3 Reus VI, Targum SD, Weingarter H, Post RM. Effect of lithium carbonate on memory process of bipolar affectively ill patients. Psychopharmacology $1979 ; 63: 39-42$.

(Accepted 21 September 1982)

Department of Psychiatry and Clinical Psychology, Southern General Hospital, Glasgow G51 4TF

ERNEST P WORRALL, MB, MRCPSYCH, consultant psychiatrist RUTH A GILLHAM, MA, MAPPSĊI, basic-grade clinical psychologist

\section{Why patients were lost from follow-up at an urban diabetic clinic}

Patients who have frequent contact with a diabetic clinic have a better prognosis than those who do not. ${ }^{1}$ We aimed to discover why patients stopped attending a diabetic clinic and what sort of supervision such patients were receiving.

\section{Methods and results}

Appointment records of the diabetic clinic from 1 January 1979 to 31 May 1980 were studied. Patients who had not attended for 13 months or more were identified, and non-attendance confirmed from the last entry in the hospital notes. If the reason for non-attendance was not apparent information was obtained from the general practitioner directly or by questionnaire.

A total of 972 patients had appointments during the study period. Fortyseven patients $(4.8 \%)$ were non-attenders: information was available on 44 $(94 \%), 13$ of whom (30\%) had in the past missed at least one appointment Two of the 44 patients had died.

Eighteen patients (12 men, six women; age range 17-78 years, mean 44) had moved to another area or clinic. Five of these patients were on a diet, two were also receiving tablets. The remaining 13 were being treated with insulin (mean dose 46 units/day): five of these patients were aged under 30 and five were in the age range 30-50. In 14 cases a further appointment was arranged. One patient was pregnant; another was admitted to hospital with self-poisoning.

Ten patients (eight men, two women; age range 18-74, mean 49) were subsequently returned to hospital care. Five were on a diet, two were also receiving tablets. The remaining five were being treated with insulin (mean 